\title{
Avoiding diagnostic pitfalls in mimics of neoplasia: the importance of a comprehensive diagnostic approach
}

\author{
Ely Zarina Samsudin ${ }^{1}$, BMedSci, BMBS, Tunku $\underline{\text { Kamarul}}^{1}$, MS, PhD, Azura Mansor $^{2}$, MS
}

\begin{abstract}
Any medical diagnosis should take a multimodal approach, especially those involving tumour-like conditions, as entities that mimic neoplasms have overlapping features and may present detrimental outcomes if they are underdiagnosed. These case reports present diagnostic pitfalls resulting from overdependence on a single diagnostic parameter for three musculoskeletal neoplasm mimics: brown tumour (BT) that was mistaken for giant cell tumour (GCT), methicillin-resistant Staphylococcus aureus osteomyelitis mistaken for osteosarcoma and a pseudoaneurysm mistaken for a soft tissue sarcoma. Literature reviews revealed five reports of BT simulating GCT, four reports of osteomyelitis mimicking osteosarcoma and five reports of a pseudoaneurysm imitating a soft tissue sarcoma. Our findings highlight the therapeutic dilemmas that arise with musculoskeletal mimics, as well as the importance of thorough investigation to distinguish mimickers from true neoplasms.
\end{abstract}

Keywords: diagnosis errors, musculoskeletal, neoplasms, tumour-like conditions

\section{INTRODUCTION}

As clinicians' diagnoses have profound impact on patients' health, it is in the patients' best interest that diagnoses are as accurate as possible. In musculoskeletal oncology, it is not uncommon to have similar presentations of different conditions in terms of clinical, radiological and histological findings. This complicates the decision of how to manage the condition since several different diagnoses need to be entertained. For example, in tumour-like conditions, a benign entity can simulate a malignant neoplasm and vice versa. To differentiate one from the other is of paramount importance, as a wrong diagnosis is potentially hazardous to the patient. The key to achieving this is familiarising oneself with such mimics and considering all diagnostic parameters before proceeding to definitive management. Herein, we present multiple instances involving tumour-like conditions where the initial diagnosis was made based solely on one outstanding diagnostic parameter. We discuss the factors contributing to these diagnostic complications with the aim of making clinicians more aware of and sensitive to the possible pitfalls in their diagnoses, thus improving their practices.

\section{CASE REPORTS}

\section{Case 1}

A 36-year-old woman who was previously healthy presented to her district hospital with a painful lump on the proximal left leg. It was first noticed two years prior to consultation and was associated with generalised myalgia and mechanical low back pain. She had no previous history of trauma, fever, cough or constitutional symptoms. Physical examination revealed a tender, hard, fixed, $5 \mathrm{~cm} \times 5 \mathrm{~cm}$ mass with no overlying skin changes on the anterior aspect of her proximal left leg. The range of motion of her left knee was limited due to pain. Examination of her back elicited tenderness at the L4 and L5 region with no neurologic signs.

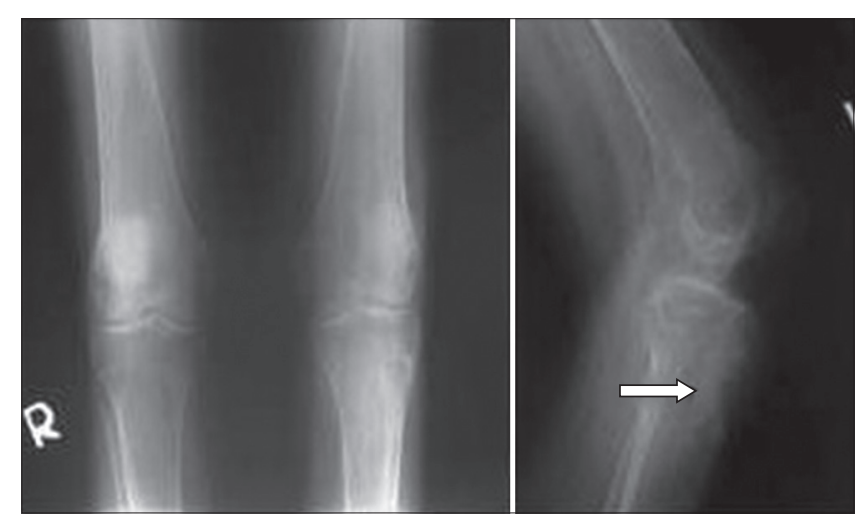

Fig. 1 Case 1. Plain radiograph of the left tibia shows a lytic lesion at the epiphysis of the proximal tibia (arrow), with the lesion poorly demonstrated on anteroposterior view.

Anteroposterior and lateral radiographic views of her left tibia and fibula performed at the same hospital showed a welldefined, eccentric, lytic epiphyseal lesion of the left tibia (Fig. 1). Spinal radiographs revealed generalised diffuse osteopenia of thoracolumbar vertebra, with no evidence of fracture. Magnetic resonance (MR) imaging of her left knee showed an enhancing, expansile mass in the anterior metaphysis of her left tibia extending to epiphyses anteriorly, with low signal intensity on T1-weighted and heterogenous intermediate intensity on T2-weighted images. The clinical impression was that she had a giant cell tumour (GCT) and she was subsequently referred for further management.

At our centre, the patient underwent a core needle biopsy of the left tibia, and the tissue sample was sent for culture and histopathological examination (HPE). Tissue HPE showed a proliferation of spindle stromal cells with nuclei features similar to those of a giant cell and osteoclastic giant cells around bony trabeculae, suggestive of a giant cell-like lesion. When we correlated the patient's history of generalised bone pain with the generalised diffuse osteopenia on her radiograph, brown

${ }^{1}$ Clinical Investigation Centre, University Malaya Medical Centre, ${ }^{2}$ National Orthopaedic Centre of Excellence for Research and Learning (NOCERAL), Department of Orthopaedics, Faculty of Medicine, University of Malaya, Kuala Lumpur, Malaysia

Correspondence: DrEly Zarina Samsudin, Medical Officer, Clinical Investigation Centre, University Malaya Medical Centre, 59100 Kuala Lumpur, Malaysia. elyzarina07@yahoo.com 


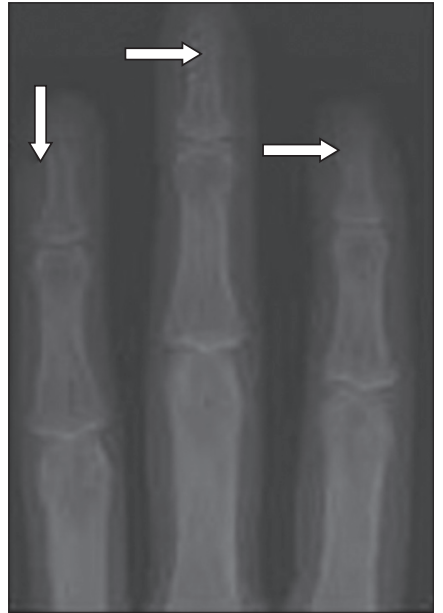

Fig. 2 Case 1. Radiograph of the hand shows phalangeal tuft resorption of the distal phalanges (arrows) of the middle and ring fingers.

tumour (BT) was added to the differential diagnosis and she was screened for hyperparathyroidism. Blood investigations revealed a high alkaline phosphatase value of 1,185 IU/L, a high calcium level of $3.13 \mathrm{mmol} / \mathrm{L}$, a low phosphate level of $0.6 \mathrm{mmol} / \mathrm{L}$ and a very high parathyroid hormone level of $163 \mathrm{pmol} / \mathrm{L}$. Further investigations were conducted to confirm BT. A skeletal survey, including a plain radiograph of the hand, showed phalangeal tuft resorption of distal phalanges but no obvious subperiosteal bone resorption (Fig. 2). Dual-energy X-ray absorptiometry indicated osteopenia with a $Z$ score of -5.5 and a $T$ score of -5.7 . Ultrasonography of the neck and 99mTc-methoxyisobutylisonitrile parathyroid scintigraphy demonstrated left parathyroid adenoma.

A diagnosis of BT secondary to primary hyperparathyroidism was made. The patient was referred to the endocrinology team for management of hyperparathyroidism and parathyroidectomy was performed. In view of an impending pathological fracture, she underwent curettage and cementation of the left tibia. Three years after the procedure, she was well with no evidence of relapse.

\section{Case 2}

A 28-year-old man presented with a two-week history of progressive pain and swelling on the left thigh, associated with intermittent fever. The pain had started off as a deep, boring pain that was worse during rest or at night and soon progressively worsened until the affected limb was unable to bear weight and the man became wheelchair-bound. He also experienced loss of appetite and weight. An anteroposterior radiograph revealed an osteolytic lesion involving the mid-shaft of the left femur, with cortex erosion and periosteal reaction (Fig. 3). Based on radiological findings, a provisional diagnosis of osteosarcoma was made and the patient was referred for further management.

Upon admission, a biopsy was scheduled. However, during detailed history-taking, it was revealed that the patient had had prolonged contact with deer carcasses following his hunting expedition in a rural forest two weeks before he had the symptoms. He claimed that his hunting partner had developed similar symptoms with similar onset. However, no further information could be obtained as his partner was uncontactable.



Fig. 3 Case 2. Plain anteroposterior radiograph shows an osteolytic lesion involving the mid-shaft of the left femur (arrows), with cortex erosion and periosteal reaction.

Otherwise, his medical history was unremarkable. Clinically, he was febrile. His left thigh was tender and warm with a deep, ill-defined swelling measuring $20 \mathrm{~cm} \times 8 \mathrm{~cm}$, but without discharging sinuses. No other skin or soft tissue lesions were noted. Movement was restricted in his left knee due to pain.

Blood investigation revealed leucocytosis $\left(17.7 \times 10^{9} / \mathrm{L}\right)$ with $86 \%$ neutrophils, and raised inflammatory markers with a C-reactive protein (CRP) level and an erythrocyte sedimentation rate (ESR) of $45.6 \mathrm{mg} / \mathrm{dL}$ and $140 \mathrm{~mm} / \mathrm{hr}$, respectively. In view of the radiographic findings, further imaging tests were ordered. MR imaging showed a lesion involving the mid-shaft of the left femur, including the surrounding circumferential soft tissue. A bone scan demonstrated isolated uptake of the left mid and distal femur. A core needle biopsy was performed and the tissue sample was sent for histopathology, culture and acid-fast bacilli tests. Both blood and bone biopsy cultures grew methicillinresistant Staphylococcus aureus (MRSA) and a diagnosis of MRSA osteomyelitis was made.

The patient was immediately commenced on intravenous (IV) vancomycin based on sensitivity results. Incision and drainage was performed for the left thigh abscess. Culture of pus also yielded MRSA. There was no other investigative evidence that pointed to malignancy or other infections, especially tuberculosis. After a total of 12 weeks of IV vancomycin and 12 weeks of oral rifampicin and fusidic acid, totalling six months of anti-MRSA therapy, ESR and CRP level were completely normalised. During subsequent months, the left thigh pain improved and he was able to ambulate. Two years after the treatment, he had regained full function of his lower left limb with no evidence of recurrence.

\section{Case 3}

A 57-year-old woman with no known medical illness was referred for a swelling of the right calf that was progressively enlarging. It was noticed two months prior to consultation. The swelling was associated with occasional throbbing pain when she walked long distances but not when she was at rest. She had no fever and no constitutional symptoms. There was also no history of recent trauma. However, five years prior to this consultation, she had sustained a closed fracture of the right tibia, which had been treated conservatively with a cast. On examination, we found a 


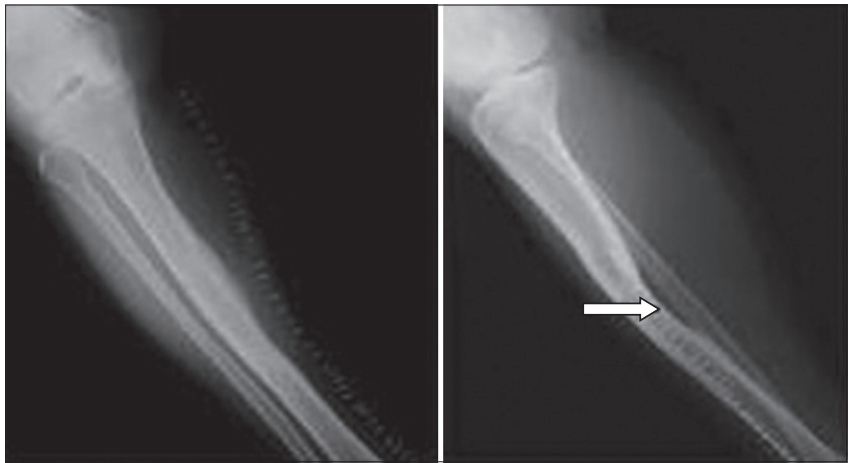

Fig. 4 Case 3. Radiograph shows scalloping of the tibia due to gradual chronic pressure of the pseudoaneurysm (postoperatively).

right calf swelling that was non-mobile, firm, warm, non-tender and measuring $10 \mathrm{~cm} \times 10 \mathrm{~cm}$. Neurovascular and systemic examinations were unremarkable.

She had initially presented to her nearest hospital, where radiography was performed, which showed a poorly defined tibial lesion with cortical erosion and scalloping of the tibia (Fig. 4). MR imaging of the right calf reportedly demonstrated a large, heterogenous and macrolobulated mass in the posterior compartment of the right calf measuring $8 \mathrm{~cm} \times 7 \mathrm{~cm} \times 10 \mathrm{~cm}$, with an irregular cortical outline and peripheral enhancement. The impression at that time was that of a large haemorrhagic mass highly suggestive of a soft tissue sarcoma, specifically, malignant fibrous histiocytoma. Computed tomography of the thorax showed no lung or liver metastasis. She was then referred to our centre for further management.

Initial core needle biopsy results at our centre were inconclusive and the patient was scheduled for an open biopsy. Intraoperative findings, however, revealed a pseudoaneurysm arising from the old tibial fracture and lacerated posterior tibial artery. Bone callous formation of the healed tibial fracture was noted to impinge on the blood vessel. The pseudoaneurysm was excised and the patient was treated with a one-week course of IV cefuroxime. Two years after the treatment, she was well with no complications or recurrence.

\section{DISCUSSION}

Clinicians are often required to make rapid decisions following a diagnosis and hence are susceptible to diagnostic pitfalls that can affect clinical outcomes. Most often, misdiagnoses occur due to overreliance on a single, often outstanding, diagnostic parameter, resulting in potential catastrophe for patients. It is therefore imperative to treat diagnoses in a multimodal way and adopt a comprehensive approach with patients, especially when tumourlike conditions further complicate the decision-making process.

In the first case, BT was initially diagnosed as GCT. As GCT and BT share many radiological and histopathological features, the misinterpretation is not surprising, especially with BT having a reported incidence of only $0.1 \%$. ${ }^{(1)}$ A literature search of the MEDLINE database revealed 17 reported cases where BT had been mistaken for either GCT, giant cell granuloma, primary malignancy or metastatic bone disease, with five cases of BT mistaken for GCT. ${ }^{(1-5)}$ Although it closely resembles malignancy,
BT is a non-neoplastic, focal manifestation of persistent hyperparathyroidism, where altered osseous metabolism is characterised by the presence of subperiosteal resorption, diffuse osteopenia and BT. Distinguishing between GCT and BT can be difficult as both usually appear as expansile, wellmarginated, osteolytic, epiphyseal lesions on plain radiographs. Histologically, both GCT and BT are composed of mononuclear stromal cells and characteristic multinucleated giant cells. The only additional feature of BT on radiographs is manifestations of hyperparathyroidism, which includes subperiosteal resorption, but they were not evident in our patient. As both imaging and HPE results were highly suggestive of GCT, a diagnosis of GCT was made. BT was only diagnosed later through biochemical testing, when the findings were correlated with a history of generalised bone pain and radiographic evidence of generalised osteopenia. Fortunately for the patient, she recovered fully after undergoing curettage and cementation. Resection of the lesion is advocated for management of $\mathrm{BT}$, as there have been cases where the condition became worse following parathyroidectomy or normalisation of parathyroid level. ${ }^{(6)}$

In the second case, the patient had MRSA osteomyelitis, but the initial clinical impression was osteosarcoma. Osteomyelitis is a non-neoplastic condition that can mimic tumours. A MEDLINE database search revealed that there were 24 reported cases of adult osteomyelitis simulating a sarcoma and vice versa to date, and four in which osteomyelitis mimicked osteosarcoma. ${ }^{(7-10)}$ This could be due to the nonspecificity of symptoms such as fever, bone pain and loss of weight, and osteomyelitis appearing malignant on imaging. On radiographs, it has an aggressive, permeated and moth-eaten appearance associated with periosteal reaction and cortical erosion. On MR imaging, it appears to extend into surrounding soft tissue and on bone scans, it demonstrates intraosseous extension. Although the imaging findings were highly suggestive of malignancy, one should take into account the patient's history of activities, especially those involving animals. In this case, the patient was heavily involved in rural deer hunting expeditions and was exposed to various possible infections. It was found that both blood and bone tissues were infected with MRSA. Fortunately, the patient recovered fully following appropriate anti-MRSA therapy.

A pseudoaneurysm, defined as a pulsating, encapsulated haematoma in communication with the lumen of a ruptured vessel, is another example of how a benign process can simulate neoplasm. Although it is relatively rare, with a prevalence of less than $3 \%$, ${ }^{(11)}$ a literature search of the MEDLINE database for all years found five reports of pseudoaneurysm mimicking a soft tissue sarcoma. ${ }^{(12-16)}$ In the third case, a premature referral for malignant fibrous histiocytoma was made based purely on the MR imaging finding that the right calf showed malignant bone lesion features, without correlating with histopathology or clinical history. Fortunately for the patient, an open biopsy was planned, where it was discovered intraoperatively that the source of the swelling was in fact a pseudoaneurysm arising from an old tibial fracture and lacerated posterior tibial artery, allowing the pseudoaneurysm to be safely resected before an impending rupture. 
In summary, our reported cases show the importance of comprehensively correlating all diagnostic parameters for a definitive diagnosis, especially in cases where there are tumourlike conditions. Such diagnostic pitfalls can be easily avoided and this will lead to improved patient care.

\section{REFERENCES}

1. Vera L, Dolcino M, Mora M, et al. Primary hyperparathyroidism diagnosed after surgical ablation of a costal mass mistaken for giant-cell bone tumor: a case report. J Med Case Rep 2011; 5:596.

2. Kao HL, Chang WC, Lee $\mathrm{CH}$, Lee HS, Huang GS. Missed brown tumors in a young adult with decreased bone density. South Med J 2010; 103:371-3.

3. Pezzillo F, Di Matteo R, Liuzza F, et al. Isolated bone lesion secondary to hyperparathyroidism: diagnostic considerations. Clin Ter 2008; 159:265-8.

4. Morano S, Cipriani R, Gabriele A, Medici F, Pantellini F. [Recurrent brown tumors as initial manifestation of primary hyperparathyroidism. An unusual presentation]. Minerva Med 2000; 91:117-22. Italian.

5. Aoune S, Khochtali H, Dahdouh C, et al. [Giant cell lesions of the maxilla disclosing primary hyperparathyroidism]. Rev Stomatol Chir Maxillofac 2000; 101:86-9. French.

6. Martínez-Gavidia EM, Bagán JV, Milián-Masanet MA, Lloria de Miguel E, Pérez-Vallés A. Highly aggressive brown tumour of the maxilla as first manifestation of primary hyperparathyroidism. Int J Oral Maxillofac Surg 2000; 29:447-9.

7. Seybold U, Talati NJ, Kizilbash Q, et al. Hematogenous osteomyelitis mimicking osteosarcoma due to Community Associated MethicillinResistant Staphylococcus aureus. Infection 2007; 35:190-3.

8. Dhanoa A, Singh VA, Mansor A, et al. Acute haematogenous communityacquired methicillin-resistant Staphylococcus aureus osteomyelitis in an adult: case report and review of literature. BMC Infect Dis 2012; 12:270.

9. Bretagne MC, Jolly A, Mouton JN, et al. [Pseudo-sarcomatous osteomyelitis in the child (author's transl)]. J Radiol Electrol Med Nucl 1977; 58:1-4. French.

10. Pezzo S, Edwards CM. Community-acquired, methicillin-resistant Staphylococcus aureus osteomyelitis secondary to a hematogenous source :case report and review. Infect Dis Clin Prac 2008; 16:398-400.

11. Norgren L. Vascular complications from limb fractures. In: Greenhalgh RM, Hollier LH, eds. Emergency Vascular Surgery. London WB Saunders, 1992: 313-30.

12. Siegel HJ, Dunham WK. Pseudoaneurysm mimicking a soft tissue sarcoma. Clin Nucl Med 2006; 31:620-1.

13. Erler K, Ozdemir MT, Oguz E, Basbozkurt M. Does false aneurysm behave like a sarcoma? Distal femoral arterial false aneurysm simulated a malign mesenchymal tumor. A case report and review of the literature. Arch Orthop Trauma Surg 2004; 124:60-3.

14. Otsuka T, Yonezawa M, Kamiyama F, Matusita Y, Matui N. Popliteal pseudoaneurysm simulating soft-tissue sarcoma complication of osteochondroma resection. Int J Clin Oncol 2001; 6:105-8.

15. Wambeek N, Munk PL, O'Connell JX, Lee MJ, Masri BA. Popliteal vascular malformation simulating a soft tissue sarcoma. Skeletal Radiol 1999; 28:532-5.

16. Boyer MI, Wang EH, Bell RS. Ruptured deep femoral artery aneurysm simulating a soft-tissue sarcoma: a case report. Can J Surg 1995; 38:92-4. 